\title{
Adult onset still's disease - case report of 2 cases
}

\begin{abstract}
Adult onset Still's disease (AOSD) is a rare disease in adults, in children also known as systemic juvenile idiopathic arthritis. We describe two patients with intermittent fevers without unknown origin. 27 years man and 75 years old woman, who presented with lymphadenopathy and recurrent fevers, There has been used intensive serologic, radiologic, laboratory investigation to exclude infectious diseases and malignancy. All the investigation showed no diagnosis. The clinical disease described for the first time 105 years ago by Dr Still is finally diagnosed. Both patients received Anakinra with rapid response in hematologic, biochemical, and cytokine markers with reduction of systemic and local inflammation.
\end{abstract}

Adult-onset Still's disease is a rare systemic inflammatory disease of unknown etiology that often presents as a fever of unknown origin. Systemic features, such as spiking fever, skin rash, generalized lymphadenopathy, hepatomegaly, splenomegaly, and serositis, was first described by the British paediatrician Still. ${ }^{1}$ The aetiology of AOSD still remains unknown, but over expression of Th1 cytokines and IL-1 may have a critical role. ${ }^{2}$

Daily fevers, evanescent rash, arthritis, hyperferritinemia, and liver dysfunction were consistent with AOSD. Hyperferritinemia has a high sensitivity for AOSD (80\%) but has a low specificity $(40 \%)$. The fraction of glycosylated ferritin is higher than with inflammatory conditions. The combination of elevated serum ferritin level and low $(20 \%)$ fraction of glycosylated ferritin can make the diagnosis of AOSD most likely. In fact AOSD has similarities to auto inflammatory diseases, as exemplified by a central role of the innate immune system and by the cytokines involved (e.g., interleukin-1 [IL-1]). Moreover, the blocking of both IL-1 has been shown to be efficacious in the treatment of AOSD. An interleukin-1 (IL-1) antagonism, e.g., with the IL-1 receptor antagonist Anakinra, is the standard of therapy for the exacerbations of acute disease rather than increasing steroid use.
Volume 5 Issue 5 - 2016

\author{
Eken Yosef, Konings Stijn, Demeyere Thomas \\ Catharina Hospital, Netherlands
}

\begin{abstract}
Correspondence: Eken Yosef, University Utrecht, Catharina Hospital, Beneluxlaan 7531363BJ almere, Netherland, Tel
\end{abstract} 31647958978 Email yusuf.eken@gmail.com

Received: July II, 2016 | Published: September 13, 2016

\section{Patient A}

\section{Chief symptom}

Fever and fatigue

\section{History of the present illness}

1. Initial Presentation April 2002.

\section{First admission 3-24 May 2002.}

A 27-year-old Caucasian male presented with an episode of fever since 4 weeks, diffuse myalgia, sore throat, cold chills and fatigue. The patient was in his usual state of health until 1 month before admission (March 2002), when he developed fever. He had an 18-pound weight loss over the preceding month. During the fever attacks he had fatigue. He did not report of sicca symptoms, arthralgia, abdominal pain, nausea, diarrhoea, cough, dyspnoea, or a rash. He denied oral ulcers, ocular inflammation, photosensitivity, or Raynaud's phenomenon. He reported no pain or stiffness in the neck, low back, or heel.

There was no history of recent travel, trauma, or sick contact. The patient's medical history was unremarkable. There was no family history of autoimmune diseases. He took no medications.

\section{Physical examination and tests}

The patient was not sick on admission. His vital signs were as follows $\mathrm{He}$ had a fever of $40.0^{\circ} \mathrm{C}\left(104^{\circ} \mathrm{F}\right)$. His blood pressure $130 / 80$ was $\mathrm{mm} \mathrm{Hg}$ and his heart rate was 102 beats /minute. There was no jugular vein distension and no murmur, gallop or rub. His abdomen was soft and not tender or distended with normal bowel sounds and no hepatosplenomegaly. His peripheral pulses were normal throughout.
There was no evidence of synovitis in any joint. There was no dactylitis, nail changes, or tenosynovitis. No purpura or petechia, no osler nodules, no exanthema, no signs of vasculitis. The remainder of the physical examination was unremarkable.

Laboratory test revealed elevated inflammatory markers, including erythrocyte sedimentation rate (ESR; $116 \mathrm{~mm} /$ hour), C-reactive protein level $(266 \mathrm{mg} / \mathrm{litre})$. A peripheral leucocytosis was present, with a white blood cell count of 22,000/mm3 (normal range 4,50011,000). WBCs, $90 \%$ neutrophils.

The serum ferritin level was markedly increased at $5696 \mathrm{mg} /$ $\mathrm{ml}$ (normal range 10-200). Urine cultures showed no infection and proteinuria was absent.

An infectious disease evaluation was performed and was negative for all pathogens, including Lyme disease, human immunodeficiency virus, cytomegalovirus, EBV. Antibodies to Sm/RNP, Ro/SSA, and $\mathrm{La} / \mathrm{SSB}$; anti cardiolipin antibodies; ant neutrophil cytoplasmic antibodies (ANCAs); and anti-cyclic citrullinated peptide antibodies were negative, and creatine phosphokinase was normal. A chest radiograph was normal. Transesophageal echocardiogram was performed by cardiologist the next day and ruled out endocarditis. (No vegetation's, some pericardial fluid).

\section{Medical history}

He only had a history of an episode of fever after a streptococcal infection ten years ago.

\section{Medications and allergies}

His medications upon admission were diclofenac $2 \mathrm{dd} 100 \mathrm{mg}$ 


\section{Diagnosis}

Adult Onset Still's Disease

\section{Subsequent clinical course}

Second Hospital admission, 12-30 December 2003, After being well during 1 year after hospital discharge he complained of since 1 month existing fatigue, diarrhea, nausea, vomiting, with fever up to $40.3^{\circ} \mathrm{C}\left(104.54^{\circ} \mathrm{F}\right)$ (Table 1$)$.

Table I Clinical features of patients

\begin{tabular}{lll}
\hline Admission & Clinical Features at Admission & Diagnosis \\
\hline First 2002 & High grade fever & AOSD \\
Second 2003 & Icteric sclera, salmon coloured rash & Liver failure by EBV \\
Third 2007 & High grade fever & Liver failure by CMV \\
\hline
\end{tabular}

He had recognized dark urine and light coloured defecations. Physical examination showed a sick patient with dry mucosa. Blood pressure 123/55, icteric skin, decreased skin turgor. Submandibular, right lateral sternocleidomastoid and right groin lymphadenopathy, all noduli seemed benign. Temperature was $38.9^{\circ} \mathrm{C}\left(102^{\circ} \mathrm{F}\right)$, the pulse rate was 122 beats/ minute, and the respiratory rate was 12 breaths/ minute. There were no signs of Raynaud's phenomenon, rash, oral ulcers, synovitis. The heart, lung examinations were normal. Abdomen is extended with normal with normal bowel sounds, splenomegaly and hepatomegaly $10 \mathrm{~cm}$ below the costal arch.

His liver function deteriorated and clotting indices were also deranged. LDH 2846 U/L GGT 319 U/L, ASAT 785U/L, ALAT 461U/L, Factor V 0.50U/ml (n 0.6-1.5), anti trombone $0.42 \mathrm{kU} / 1$ (n $0.8-1.15$ ), ammoniac $84 \mu \mathrm{mol} / \mathrm{L}$ (n 16-35), total Bilirubin $261 \mu \mathrm{mol} / \mathrm{L}$, Ferritin $>16500 \mu \mathrm{g} / \mathrm{L}, \mathrm{CRP} 156 \mathrm{mg} / \mathrm{L}$, Sodium $119 \mathrm{mmol} / \mathrm{L}$, Potassium $3.1 \mathrm{mmol} / \mathrm{L}$.

Extensive evaluation of his fevers, including routine blood, urine, and sputum cultures as well as viral and fungal serology, determined an infection with EBV. Echo abdomen showed normal blood flow in liver en spleen. The patient developed severe salmon pink coloured maculo papullary rash over his face, upper arms and on his back. He had an acute liver failure due to EBV infection.

Prednisone $60 \mathrm{mg} /$ day was started. The patient's localized rash and fever resolved spontaneously. Liver function showed improvement. He was discharged from our hospital after 3 weeks

Reactivation AOSD with liver function disorders: Liver biopsy June 2004 (Figure 1): Chronic non specific hepatitis persisted. He received after discharge 1.5 year sucralfate for hyperbilirubinemia. Prednisone is continued in low dose at discharge until May 2006 than stopped. Methotrexate (MTX) therapy was started. The weekly dose was increased over 9 months to $25 \mathrm{mg}$.

Third hospital admission, 28 March- 18 April 2007: He was admitted to our hospital (his third) for recurrent high-grade fever. He had 1 week before presentation to our centre, the patient developed intermittent fevers, and severe proximal lower extremity myalgia associated with weakness, an erythematous rash on his upper extremities. Laboratory evaluation showed elevated liver enzymes and trombocytopeny.

Trombocyts 99/nL, CRP $81 \mathrm{mg} / \mathrm{L}$, BSE 5mm/hour, ASAT 585U/L, LD 886U/L, AF 264, IU/L GGT 523U/L, bilirubin $60 / 50 \mu \mathrm{mol} / \mathrm{L}$ ferritin $>16500$ ).

Extensive evaluation of his fevers, including routine blood, urine, and sputum cultures as well as viral and fungal serology, determined an infection with CMV. He had another relapse AOSD because of a CMV infection. Computed tomography (CT) of the thorax and abdomen was performed to evaluate his fever and weakness. There was no evidence of abscess, or malignancy. The patient underwent a liver biopsy that showed CMV hepatitis (Figure 1). The patient was treated empirically with intravenous ganacyclovir and cymevene.

He was treated with Prednison in high dose.

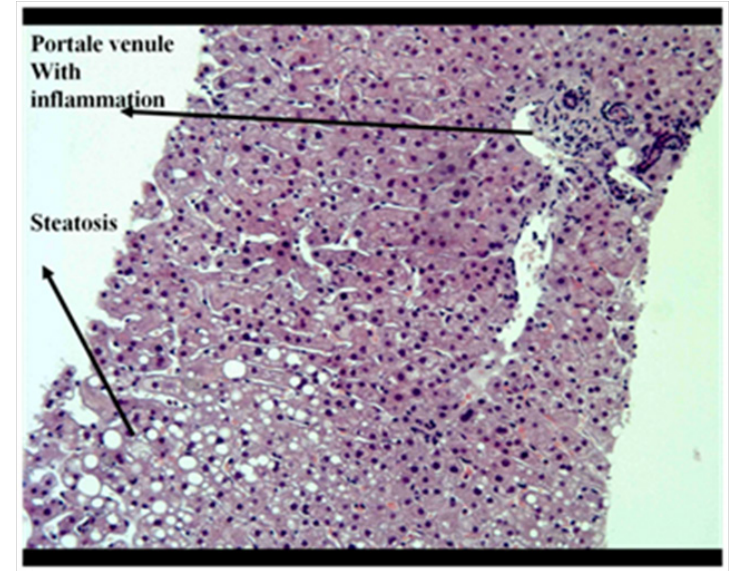

Figure I Patient A liver biopsy 2004.

March 2009 before start anakinra: Liver functions showed elevated enzymes during Methotrexate and Prednison therapy. CRP $14 \mathrm{mg} / \mathrm{L}$, ferritin $1095 \mu \mathrm{g} / \mathrm{L}$, total bilirubin $72 \mu \mathrm{mol} / \mathrm{L}$, conjugated bilirubin $46 \mu \mathrm{mol} / \mathrm{L}, \quad$ ASAT850U/L, ALAT1500U/L, LD420U/L, alkalic phosphates 491IU/L gamma GT410U/L. Methotrexate has been continued until Anakinra was started

November 2009 after start Anakinra: He received Anakinra $1 \mathrm{dd} 100 \mathrm{mg}$ as monotherapy in October 2009 with improvement of systemic inflammation and fever attacks. Liver function is normal. Laboratory investigation showed bilirubin total $30 \mu \mathrm{mol} / \mathrm{L}$, ASAT 22U/L, ALAT 29U/L, LD 170U/L, alkalic phosphates 121IU/L, CRP $<6.0 \mathrm{mg} / \mathrm{L}$. Since start of the treatment with Anakinra he had no complaints or had any relapse.

\section{Patient B}

\section{History of the present illness}

75 years old women presented at our hospital with since months daily fevers between 38 to $39.5^{\circ} \mathrm{C}\left(100.4^{\circ}-103.1^{\circ} \mathrm{F}\right)$ with night sweats. Her complaints were of fatigue, dizziness and nausea. Besides the febrile episode she felt well. Attacks of fever occurred sometimes 2 times a day, some days she had 3 days following without fever. She lost 10 kilograms in 1 month. She had no recent tick bites or other infections. She was recent back from holiday in France. During holiday and before she had no contact with domestic animals, no tuberculosis contact, no erythematic rash, no photosensitivity, no Raynaud's phenomenon, no ulcers, no stiffness over the muscles of the legs and arms, no visual or hearing problems. No jaw claudication and no arthritis. There was no family history of autoimmune disease. She took no medications.

\section{Medical history}

Diabetes Mellitus type II, atrium flutter

\section{Medications and allergies}

Atenolol/chloortalidon 1dd100/25 mg, Metformine 2dd500 mg 


\section{Physical examination and tests}

Physical examination revealed fully alert, oriented, and lucid sick women. Her vitals sign were as follows. Blood pressure 150/80 $\mathrm{mm} \mathrm{Hg}$, Heart beat 76 /minute, her oxygen saturation was $95 \%$ on room air, and Temperature $37.8^{\circ} \mathrm{C}\left(100^{\circ} \mathrm{F}\right)$, her respiratory rate was 18 breaths/ minute un labored. Unilateral right sided supraclaviculair lymphadenopathy with Right thyroid gland nodule. Examination of her heart revealed an irregular tachycardia (atrial flutter on ECG) but no murmurs, rubs, or gallops. The lungs were clear to auscultation. There was no evidence of hepatosplenomegaly, skin rash, focal motor weakness, sensory deficits or arthritis. Laboratory examination showed elevated inflammation levels (CRP $133 \mathrm{mg} / \mathrm{L}$, BSE 48mm/h). Leucocytes $13.1 / \mathrm{nL}$ Neutrophils $11 / \mathrm{nL}$, Calcitonin lightly elevated. No M protein detectable, ACE not elevated. Assays for antinuclear antibodies, antibodies to double stranded DNA, rheumatoid factor, anti-neutrophil, cytoplasmic antibodies, anti-phospholipid antibodies, and cryoglobulins were negative. The serum $\mathrm{C} 3$ and $\mathrm{C} 4$ levels were normal. A serum protein electrophoresis test showed normal levels of all immunoglobulin and no monoclonal spike. Blood cultures and a tuberculin test were negative. ECG: Atrium flutter. A complete workup, including lymph node, bone marrow biopsy, was done to exclude malignancy. Her blood, stool, urine cultures and viral serology were all negative. All serologic examination was negative. Chest radiogram showed cardiomegaly. An ultrasound scan of the abdomen showed no abnormalities.

1. CT thorax: Multiple pathologic mediastina lymphomas (Figure 2).

2. Pet scan: Elevated uptake in multiple pathologic nodule high supraclavicular rightand mediastina.

3. Mediastinal lymph nodule biopsy: No malignant cells. Nonspecific reactive Para cortical hyperplasia.

4. Biopsy middle lung: No malignancy.

5. Cytological punction thyroid gland nodule: Normal.

6. Bronchoscopy: No tumour.

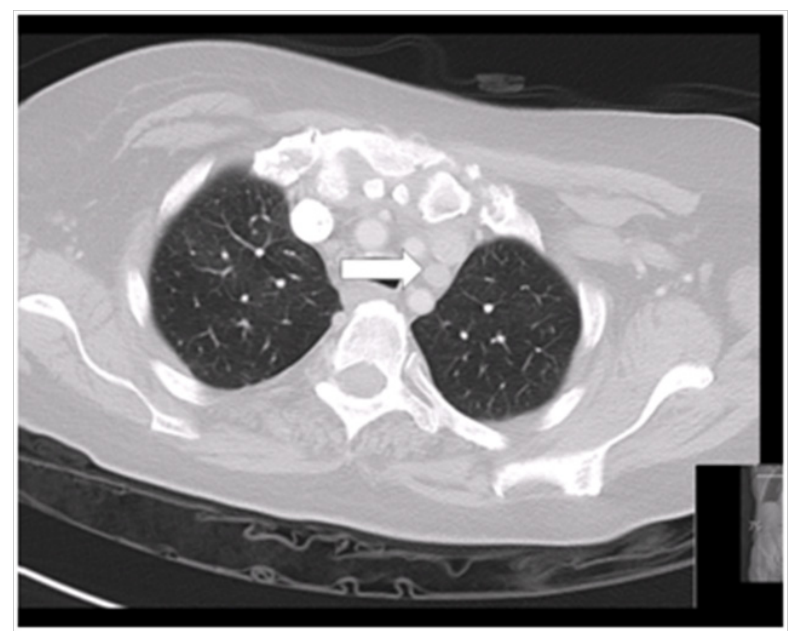

Figure 2 Patient B Ct thorax September 2010.

\section{Hospital course}

She had daily fever attacks with hypotension during febrile episode During admission of 7 weeks there were 2 periods of $4-5$ days without fever. Most of the febrile attacks occurred at night or early in the morning. At the beginning of the hospital stay were the levels of fever higher than compared with the last weeks. She developed during 2nd week pericarditis. Except for the fever attacks she was hemodynamic stabile; only during a febrile attack she had hypotension and fatigue. She received Anakinra with clinical remission. There was an initial response without relapse.

\section{General discussion}

These case reports illustrate that we have to be carefully to assume a diagnosis of AOSD. There is no laboratory examination or histological findings that can be diagnostic for, which is purely a clinical diagnosis. Exclusion of malignancies of the lymphoreticular system is very important in establishing the diagnosis. Our patient's intermittent fevers to greater than $38.3^{\circ} \mathrm{C}$ for more than 3 weeks meet the classic definition of fever of unknown origin (FUO). Reviews of large case series of FUO suggest that a primary inflammatory process is responsible for the fever in $22 \%$ of cases. Infection and malignancy account for $16 \%$ and $7 \%$, respectively, and miscellaneous conditions account for an additional $16 \%$. More than half of cases of FUO have remained without diagnoses in some series.

High fever, rash, liver dysfunction, ferritin can be consistent with sepsis, Endocarditis should also be considered. Both patients thought to have an infection as a most probable cause of fever. Serologic examination revealed no infectious cause. In both patients Cardiologist excluded an endocarditis. Patient A had no lymphadenopathy at presentation. Patient B had a few nodules supraclavicular. After radiological investigation there were multiple mediastinal nodules by patient B.

Malignancy especially Lymphoma and leukaemia are excluded by biopsy.

\section{Pathologic discussion}

Dr. Demeyere T, The biopsy of the liver and lymph nodule biopsy showed no typical sign of malignancy, tuberculosis infection, other infectious cause, sarcoidosis or Kikuchi disease. The only finding at histological examination was nonspecific reactive Para cortical hyperplasia. There was no necrosis and only slight inflammation. Medistinal biopsy and lung biopsy of patient B was negative for malignancy.

\section{Final diagnosis}

Adult Onset Still's Disease.

\section{Acknowledgments}

None.

\section{Conflicts of interest}

None.

\section{References}

1. Still GF on a form of chronic joint disease in children. Med Chir Trans. 1897:80:47-60.

2. Efthimiou P, Paik PK, Bielory L Diagnosis and management of adult onset Still's disease. Ann Rheum Dis. 2006:65(5):564-572. 